\title{
La Contribution Du Port De Pêche Á La Dynamique De La Distribution Spatiale Des Ressources Halieutiques Á Abidjan
}

\author{
Aka Koffi Sosthène, \\ N'cho Amalatchy Jacqueline, \\ Docteur en géographie, chargé de recherche au Centre de Recherches \\ Océanologiques (C.R.O.), Abidjan, Côte d'Ivoire \\ Kouman Koffi Mouroufié, \\ Maître de conférence à l'Ecole Normale Supérieure (E.N.S.) Abidjan \\ Université Félix Houphouët Boigny de Cocody-Côte d'Ivoire
}

Doi:10.19044/esj.2019.v15n36p305 URL:http://dx.doi.org/10.19044/esj.2019.v15n36p305

\section{Resume}

La construction d'un port en eau profonde a été effective en 1951, suite au percement du canal de Vridi. Il s'est agrandi au fil du temps. Cet instrument primordial, mieux outillé dans l'import-export connaît un démembrement en plusieurs secteurs d'activités dont celui du port de pêche. Cette entité portuaire, quoiqu'embryonnaire en 1963, s'est améliorée et est devenue aujourd'hui l'indispensable pourvoyeur en ressources halieutiques de la ville d'Abidjan et de son hinterland. L'objectif de cette étude est d'analyser le rôle du port de pêche dans la distribution spatiale des produits halieutiques à Abidjan. Ce facteur est essentiel eu égard aux nombreuses sollicitations des différents opérateurs économiques œuvrant dans ce secteur d'activité. L'augmentation de la population de cette cité qui se chiffre à 4.707 .000 habitants (I.N.S, 2014) est source d'une consommation accrue de ces denrées riches en protéines animales et à la portée des bourses. Quel dynamisme présente le port de pêche dans la distribution des ressources halieutiques à Abidjan. La revue de littérature et les enquêtes de terrain ont été des méthodes privilégiées pour l'obtention des informations relatives à son fonctionnement, à son organisation auprès des différents acteurs de la filière. Les résultats affichent un dynamisme dans le mode de fonctionnement et dans la distribution qui part des grossistes aux petits détaillants. Cet état de fait lui donne une coloration particulière, élément de structuration spatiale par excellence à travers ces différents relais que sont les chambres froides, les marchés. Des faiblesses subsistent toutefois dans cette vitalité de distribution qu'il convient d'appréhender. Des réseaux de production et de distribution annexes viennent en appoint pour une occupation rationnelle du marché aux 
fins de combler la faiblesse du circuit et de satisfaire la demande des populations.

Mot clés: Côte d'Ivoire, Abidjan, Dynamisme, Port de pêche, Structuration

\title{
The Contribution of Fishing Port to the Dynamics of the Spatial Distribution of Fisheries Resources in Abidjan
}

\author{
Aka Koffi Sosthene, \\ N'cho Amalatchy Jacqueline,
}

Docteur en géographie, chargé de recherche au Centre de Recherches

Océanologiques (C.R.O.), Abidjan, Côte d'Ivoire

Kouman Koffi Mouroufie,

maître de conférence à l'Ecole Normale Supérieure (E.N.S.) Abidjan

Université Félix Houphouët Boigny de Cocody-Côte d'Ivoire

\section{Abstract}

The construction of a deep port was effective in 1951, following the piercing of the Vridi canal. It has grown over time. This primordial instrument, better equipped in the import-export sector, is dismembered into several sectors of activity including that of the fishing port. This portal entity, although embryonic in 1963, has improved and has become today the indispensable provider of fishery resources in the city of Abidjan and its hinterland. The objective of this study is to analyze the role of the fishing port in the spatial distribution of fish products in Abidjan. This is essential in view of the numerous demands made by the various economic operators and the increased consumption of these foodstuffs, which are rich in animal protein, due to the population increase of 4707000 inhabitants (INS, 2014). What dynamism presents the fishing port in the contribution of fishery resources to Abidjan. The literature review and field surveys have been preferred methods for obtaining information on its functioning, its organization with the various players in the sector. The results show a dynamism in the mode of operation and distribution from wholesalers to small retailers. This state of affairs gives it a particular coloring, an element of spatial structuring par excellence through these various relays as cold rooms, markets. Weaknesses remain, however, in this vitality of distribution that should be apprehended. Additional production 
and distribution networks add to the rational use of the market. Tangible proposals can be made.

Keyword: Côte d'Ivoire, Abidjan, Dynamism, Fishing port, Structuring

\section{Introduction}

Le port est un lieu aménagé sur le littoral maritime, sur les berges d'un lac ou en certains endroits d'un important cours d'eau. C'est donc une infrastructure construite par l'homme pour recevoir et abriter les navires et leur permettre de charger et de décharger ce qu'ils transportent (CNRLTL, 2012). Celui de la Côte d'Ivoire est bâti en eau profonde sur la lagune Ebrié et sa gestion est assurée par un conseil d'administration réunissant plusieurs partenaires. Le port est la porte d'entrée et de sortie des personnes, des marchandises et des biens. Cette ouverture sur le monde extérieur est synonyme d'échanges d'envergures à tous égards. Sa performance est le reflet économique d'un pays. Celui de la Côte d'Ivoire est une entité à l'intérieur de laquelle se dégagent des spécificités dont celle de la pêche.

Ici, les ressources halieutiques constituent la matière première de ce démembrement portuaire, largement dominées par le poisson dans toute sa composante. La faible production annuelle de 50294 tonnes (DAP, 2012) et l'augmentation de la population abidjanaise qui s'évalue à 4707000 habitants (I.N.S., 2014) riment avec une importation massive qui atteint $260800 t$ (DAP, 2012). Il faut donc desservir Abidjan et son hinterland en produit halieutique pour sécuriser les populations en protéines animales par le biais du port de pêche. La fonction portuaire est un important facteur de régularité dans l'organisation spatiale des villes en termes de morphologie interne et de profil fonctionnel (DUCRUET, 2005).

Le port de pêche à certes connu des mutations, des aménagements pour remplir pleinement sa fonction qui lui est dévolue. Face à l'évolution accélérée de la population abidjanaise, le port arrive-t-il à desservir convenablement toute la cité ? Autrement dit, quel est le rôle du port de pêche dans la distribution des produits halieutiques dans l'espace abidjanais ? N'existe-t-il pas d'autres filières annexes qui participent aux besoins des populations? De façon spécifique, ce travail consiste à analyser la dynamique du port de pêche dans son rôle d'organisation et de distribution des produits halieutique dans l'espace abidjanais.

La méthodologie de travail adoptée pour y parvenir est la revue bibliographique, suivie d'une enquête de terrain soutenue.

La première phase correspond à un passage en revue des périodiques, des documents relatifs au port de pêche. Toutefois les statistiques relevant du domaine des pêches ont été feuilletées tant au niveau des espèces capturées que des importations. Aussi, pour élargie le champ de connaissance fallait-il 
explorer d'autres horizons pour une nécessité de comparaison et d'approfondissement de certaines notions.

L'enquête de terrain qui constitue la seconde phase est faite d'observations directes, essentielles pour la consolidation des acquis, d'un questionnaire structuré ou semi-structuré. Les interviewes ou entretiens viennent en complément.

Ces différentes composantes ont été administrées à des personnes ressources du port de pêche que sont les administrateurs, les opérateurs économiques de la filière pêche ayant un lien avec l'état des stocks des ressources halieutiques, les statistiques du poisson commercialisé. L'apport des acteurs de la commercialisation (grossistes, demi-grossistes, détaillants), des armateurs a été utile. À cette gamme d'intervenants, il était essentiel de découvrir le mode de sécurisation.

L’organisation de la distribution a été mieux appréhendée par la méthode de suivi d'itinéraire des véhicules frigorifiés dans le cadre du ravitaillement des chambres froides d'Abidjan. Toute cette méthode pour mieux cerner, découvrir l'importance du mode de distribution et l'emprise spatiale des produits halieutiques du port de pêche à Abidjan. Nonobstant, la visite d'autres circuits annexes de diffusion en dehors de l'espace portuaire a été faite pour leur complémentarité dans la desserte de la ville.

Les données recueillies ont été traitées à l'aide d'outils appropriés que sont les logiciels Word et Adobe Illustrator 11.1. L'enquête a connu une durée d'un mois allant du 29 Juillet au 30 avril 2016. Le non-respect du calendrier préétabli de commun accord avec les autorités a constitué un obstacle majeur. Ce devoir peut être charpenté autour de 3 principales parties :

- $\quad$ Présentation du port de pêche et son système organisationnel

- $\quad$ Les captures et l'organisation du mode de distribution

- $\quad$ les apports extérieurs au port de pêche dans le circuit de distribution des produits halieutiques.

\section{I - Presentation du port de peche et son systeme organisationnel \\ 1. Présentation du port de pêche}

Le port de pêche d'Abidjan est situé en eau profonde, en lagune Ebrié dont l'accès à la mer se fait par l'entremise du canal de Vridi. De renommée internationale du fait d'un important flux, il s'étend sur une superficie de $200000 \mathrm{~m}^{2}$ de terre-plein sous douane et $800.000 \mathrm{~m}^{2}$ hors douane avec 1522 mètres linéaire de quai. Il est localisé au Sud du pays à $4^{\circ} 20$ latitude nord et $5^{\circ}$ longitude ouest comme l'indique la figure 1 . 
Figure 1 : Localisation du port de pêche d'Abidjan

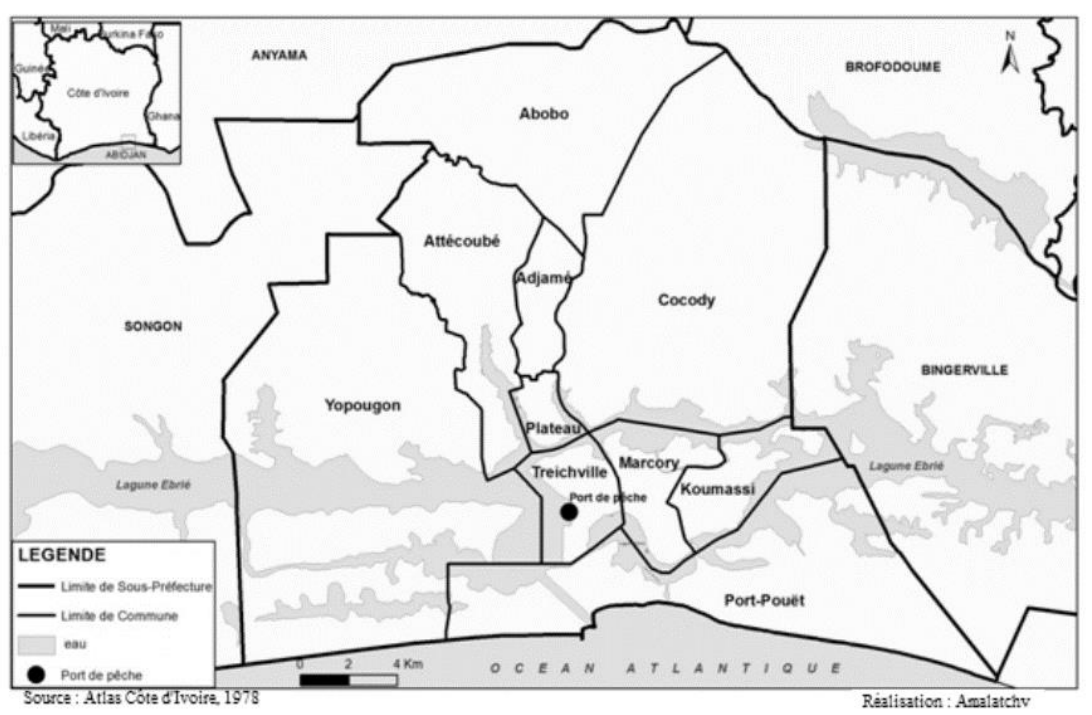

Le débouché en lagune est stratégique pour faciliter les opérations, eu égard aux nombreuses houles et marées qui pourraient déranger l'accostage des navires en mer. Avec cette façade maritime de $560 \mathrm{~km}$ de long, les avantages sont énormes pour créer plusieurs ouvrages de ce type. L'existence d'un grand port de pêche s'impose à ce pays pour le ravitaillement d'Abidjan et de son hinterland en ressources halieutiques. Les mutations, les adaptations, facteurs de dynamisme d'un port font partie de la planification au niveau de la pêche et de la navigation.

Dans le souci d'une organisation et d'une meilleure distribution des ressources halieutiques, la Côte d'Ivoire s'est dotée d'un premier port de pêche à Abidjan en 1963 avec auparavant une mise en place provisoire d'un quai construit en 1955 sur lequel étaient installés des hangars. L'ascension véritable débute en 1980 avec une amélioration des infrastructures existantes. De ce constat, l'on assiste à une création de richesse basée sur les investissements et tous les emplois générés (travailleurs permanents, saisonniers ).

\section{Administration ou système organisationnel, un moyen efficient de réussite}

Chaque secteur portuaire est autonome à l'image de sa dénomination "Port Autonome d'Abidjan". Le port est autonome lorsque sa gestion est non assurée par un directeur relevant du ministère, mais par un conseil d'administration réunissant les représentants de la municipalité, de la chambre de commerce, des syndicats, (CNRLTL, 2012). Ce mode de fonctionnement nécessite un climat favorable soutenu par la création d'un forum de rencontres et d'échanges permanents entre les membres. Il existe à cet effet, plusieurs 
logiques de gestion de l'espace, laquelle doit être effectuée pour le bien-être de l'homme (Camara, 2008). Le système organisationnel repose sur les hommes à travers une administration évolutive dans le Temps. Du statut de la Direction des Opérations Maritimes et de Sécurité (DOMS), l'on a transité par la Direction des Exploitations (DE) pour être érigé en Direction de Port de Pêche (DPP) (PAA, 2002). Ce patrimoine se compose de plusieurs services pour assurer une meilleure gestion, un meilleur suivi, une sécurité renforcée comme le montre la figure 2. 


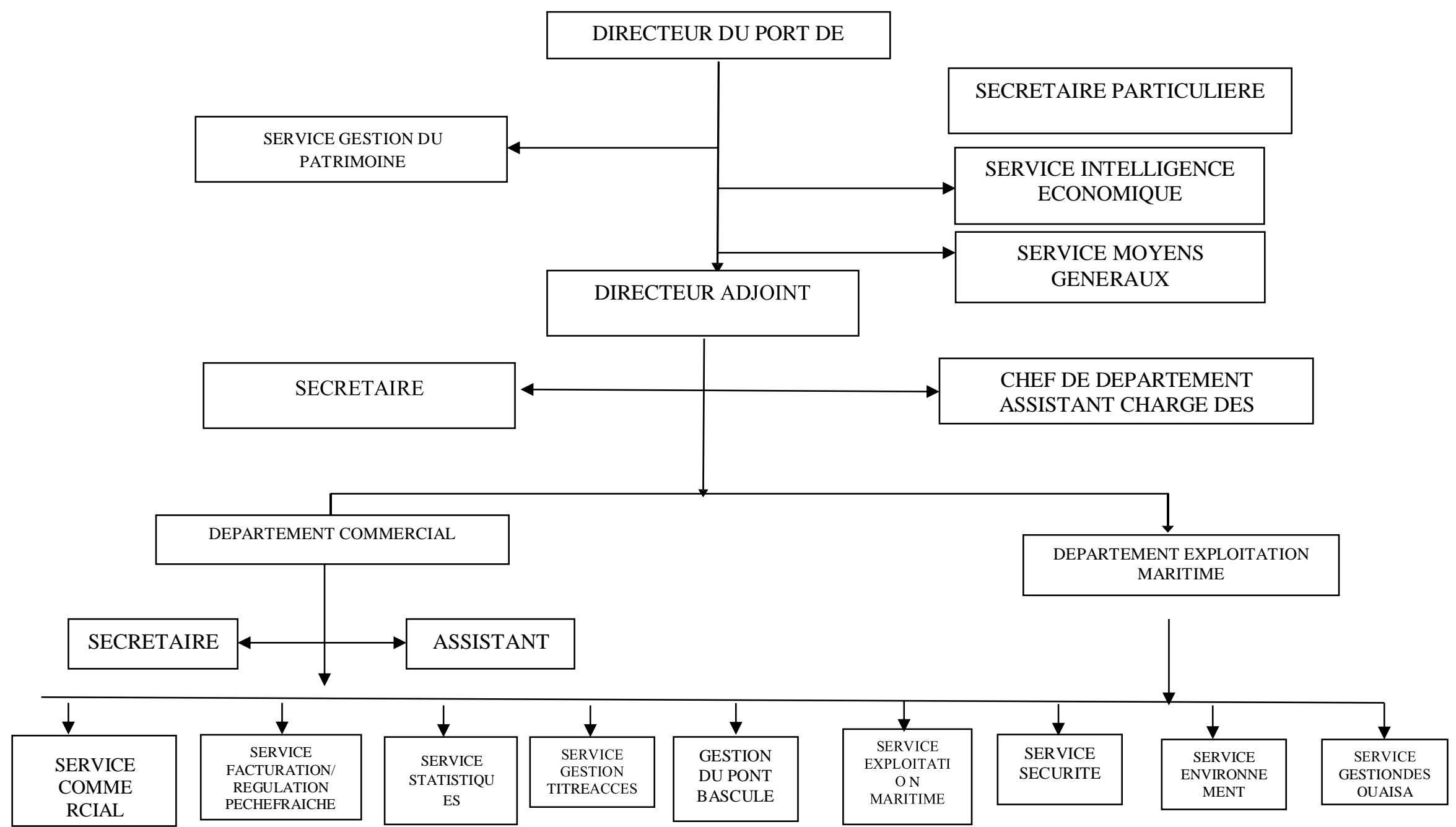


Abidjan entrevoit 29 milliards pour les travaux d'extension et de réhabilitation. L'expérience québécois a consisté à injecter des capitaux nécessaires, à mettre en place un vaste programme de construction navale afin de rendre les industries rentables et d'améliorer le bien-être des gens (Laurin, 1970)

La direction du port de pêche ivoirien a quant à elle pour mission d'accueillir des navires de pêche et de favoriser le transit des produits de pêche dans les conditions optimales de sécurité et de célérité. En outre, elle doit coordonner les activités des différents intervenants du port de pêche, garantir le suivi mis en œuvre, la politique commerciale tour en prenant en compte le facteur environnemental et le maintien en état des infrastructures. La gestion de l'accès au port et l'application de la règlementation sont en vigueur.

La matière première est le poisson et les autres produits halieutiques. Quelles sont les espèces qui inondent les quais et quel est le mode de répartition?

\section{II- Les Captures Et Organisation Du Mode De Distribution \\ 1. L'évaluation de volume de stock}

Les espèces capturées par la flotte industrielle sont classées selon le type de pêche. Il y a d'une part, les espèces produites par les chalutiers et les sardiniers et d'autre part celles fournies par les thoniers. Hormis le thon qui fait l'objet d'une pêche internationale, les autres produits halieutiques au niveau du poisson les plus pêchés et vendus de 2007 à 2014 sont les suivants : les espèces démersales sont les brochets (Sphyraena piscatorum), les capitaines (Galéoïde decadactylus), les carpes blanches (Pomadasys jubelini), les grondins (Triga sp), les loches (Brotula barbatua), l'ombrine (Pseuddotolithus senegalensis), les pageots (Pagellus coupei) les raies ordinaires (Rhinobatos sp), la sole (Cynoglassus canariensis). Il faut également souligner une abondance des espèces pélagiques dominées par le chinchard (Decapterus punctatus), la friture ordinaire (Brackydeuterus auritus), le hareng (Sardinella maderensis), le maquereau (scomber japonicus), la sardine (sardinella aurita) (PAA, 2014). La représentativité des crevettes et des autres ressources est infime (environ 1/100)

L'évolution cumulée des produits de pêche de 2010 à 2012 se présente comme suite avec une partie active de la pêche industrielle tenant compte des importations. 
Tableau 1 : L'évolution cumulée des produits de pêche au port de pêche d'Abidjan de 2010 à 2012

\begin{tabular}{|l|l|l|l|}
\hline Années & $\mathbf{2 0 1 0}$ & $\mathbf{2 0 1 1}$ & $\mathbf{2 0 1 2}$ \\
\hline Le cumul des pêches + importations (tonnes) & 644671 & 583529 & 603959 \\
\hline Pêche industrielle + importations (tonnes) & 611324 & 550363 & 553376 \\
\hline
\end{tabular}

Source PAA, 2014

A l'analyse du tableau, le flux de ressources halieutiques est essentiel. Ces denrées transitent par le port d'Abidjan où certaines espèces comme le thon subissent des transformations au plan industriel (1/3). La seule ville d'Abidjan consomme le 1/3 des ressources de pêche. Cet état de fait confirme le dynamisme du port de pêche d'Abidjan dans son rôle économique essentiel (importations, exportations).

De façon générale, la fonction portuaire est un important facteur de régulation dans l'organisation spatiale des villes, en termes de morphologie interne et de profil fonctionnel. Elle instaure une relation paradoxale entre dynamisme urbaine et mobilité de flux (DUCRUET, 2005). Aussi, la ville peut être considérée comme le moteur de l'expansion portuaire (VERLAQUE, 1979). Une corrélation existe donc entre l'évolution de la population citadine et la consommation des ressources halieutiques au vu des besoins en ressources halieutiques. Abidjan a connu une véritable croissance de sa population de 951216 habitants en 1975, elle est passée à 4707000 en 2014 (INS, 1975,2014). La production nationale est comptabilisée en dehors du thon qui fait l'objet d'une pêcherie internationale. La part nationale est de 50 294 tonnes avec une pêche maritime de l'ordre de 29754 tonnes pour une consommation de 13,92 kg/hbt/an (DAP, 2012). Il faut donc garantir la sécurité alimentaire. Il convient également de découvrir les véritables activités développées et le mode de distribution.

\section{Organisation du mode de distribution}

La distribution des produits halieutiques dans la ville d'Abidjan est un vaste processus avec en amont le port de pêche. Son organisation faite d'hommes et de femmes permet quoique difficile de desservir cette ville et ses environs. Les acteurs de la commercialisation sont les mareyeurs grossistes, les demi-grossistes, les détaillants, les transporteurs. Tout ce dispositif pour pallier au problème de protéines animales en ressources halieutiques à travers les différents marchés, considérés comme les espaces privilégiés. Il s'agit d'une chaîne qui connaît un ajout de valeur marchande à chaque étape de la vente. 


\subsection{Les mareyeurs grossistes organisés en établissement en 2015}

C'est un groupe dynamique de 75 personnes. Elles ont toutes des agréments leur permettant de mener à souhait leurs activités. Seulement une quinzaine arrivent à pratiquer convenablement cette tâche.

Ce nombre réduit s'explique par le fait que ce milieu est difficile d'accès et l'intégration des nouveaux arrivants est pénible au risque de ravir les marchés des anciens du métier. Les grossistes occupent des espaces mieux structurés avec des bureaux et du personnel mieux outillé. Chaque établissement de mareyeurs grossistes emplois en moyenne 10 à 15 personnes de façon régulière.

\subsection{Les mareyeurs demi-grossistes, un maillon défaillant de la chaîne de commercialisation}

Ils sont moins nombreux, 27 au titre de l'année 2015 à cause d'une certaine instabilité. Ils sont également organisés en établissement et servent d'intermédiaires entre les grossistes et les détaillants. Ils prennent donc les produits halieutiques dominés par le poisson chez les grossistes pour les revendre aux détaillants.

Ce maillon de la chaîne de commercialisation est souvent défaillant pour diverses raisons. L'activité est périodique et les acteurs de ce groupe sont souvent incapables d'acheter la totalité des marchandises des grossistes. Dans ces conditions, les grossistes sont contraints d'écouler la totalité de leurs marchandises en s'adressant directement aux détaillants. La période d'abondance correspond à une marge bénéficiaire faible chez les demigrossistes.

\subsection{Les détaillants}

Les détaillants sont les marchands qui s'approvisionnent auprès des demi-grossistes et doués dans la vente au détail. Ils sont les plus nombreux dans la chaines de distribution et n'ont pas besoin d'agréments pour exercer. Ils se présentent sous diverses formes et ont pour plateforme de vente, le marché situé en zone hors douane. Ils sont très actifs dans la diffusion des produits halieutiques dans la cité et hors. Certains parmi eux font la location des conteneurs frigorifiés déjà installés pour les opérations de vente; d'autres en disposent déjà installés dans les différentes communes du district d'Abidjan qu'ils approvisionnent directement du port. De ce constat, l'on dénombre deux types de détaillants : ceux des locations de conteneurs et ceux qui livrent directement leurs produits à la grande population consommatrice.

* Les détaillants locataires de conteneurs frigorifiés

21 conteneurs frigorifiés ont été identifiés dans le marché du poisson du port. Le faux thon est l'espèce essentielle. Chaque conteneur est créateur d'emplois 
avec 5 personnes pour la vente. Les ressortissants burkinabés et nigériens dominent ce marché.

* Les détaillants directs

Ils sont regroupés au sein d'une association dénommée ACAMPI (Association Coopérative des Auxiliaires Mareyeurs de la Pêche Industrielle). Elle comporte 101 personnes dont 4 femmes avec $40 \%$ de nationaux. Ils ont pour rôle l'achat et la revente du poisson. Toute cette gamme d'activité ne peut aboutir sans les moyens de transport adéquats.

\subsection{Les transporteurs}

Ils assurent la distribution du poisson entre le port de pêche et les centres de distribution. Il est dominé par les véhicules benne. Les transporteurs sont protégés au sein du syndicat National des Transporteurs de Marchandises, Voyageurs de Côte d'Ivoire (SNTMVCI), section port de pêche. Chaque véhicule est autorisé à travailler avec 4 chargeurs. 30 véhicules jouent ce rôle jusqu'au lieu de grande distribution, c'est-à-dire les marchés de poissons en zone hors douane. A la demande des clients, le poisson est conduit en dehors de la plateforme portuaire à destination des communes d'Abidjan ou des villes périphériques comme Grand-Bassam, Dabou, Bingerville...). Les chambres frigorifiées des communes servent de relais auprès desquelles les femmes viennent s'y approvisionner pour le marché. Cela amène à établir la carte de densité des poissonneries par communes (figure 3)

Figure 3 : Densité des poissonneries par commune dans la ville d'Abidjan

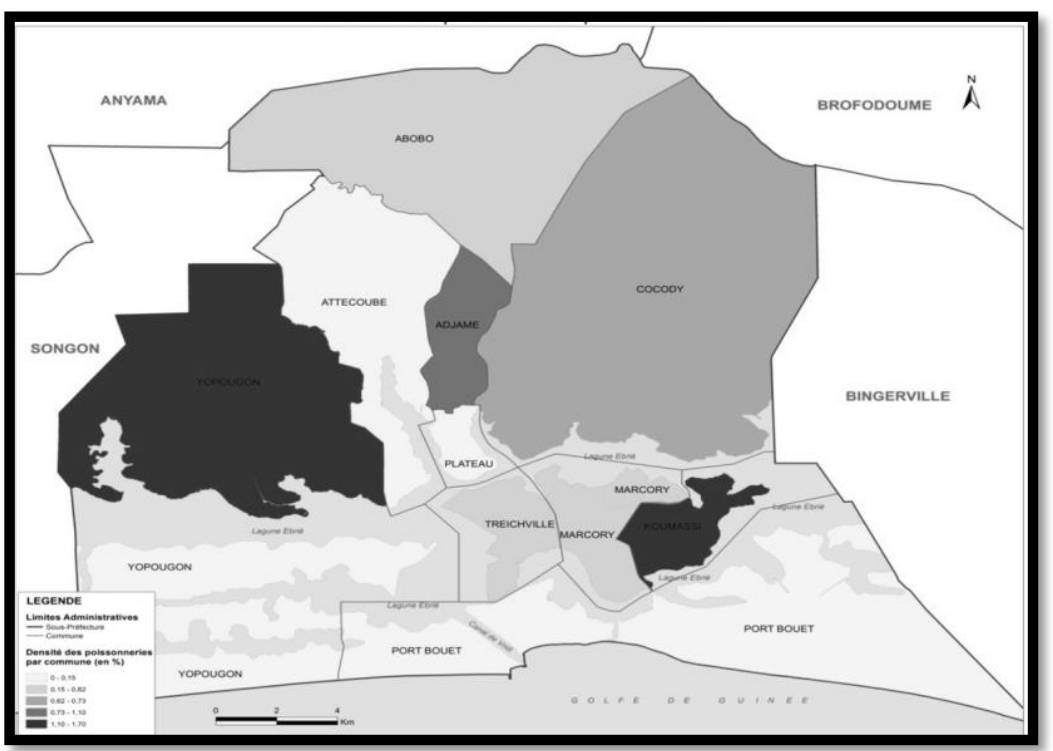


Le poisson du port est mieux structuré dans la distribution. Cependant d'autres acteurs annexes participent au ravitaillement de la ville en itchyofaune, montrant du coup des insuffisances.

\section{Les Apports Exterieurs Au Port De Pêche Dans Le Circuit De Distribution Des Produits Halieutiques}

Le ravitaillement en produits halieutiques de la ville d'Abidjan connaît un autre circuit de distribution en marge de celui du port. Ces ressources halieutiques proviennent des fleuves, des lagunes, des lacs assortis des barrages hydroélectriques et de la mer pour l'essentiel.

1. Les acteurs et les espèces dominantes

Ce circuit annexe de distribution est animé par les hommes (Nigériens, Burkinabé, Maliens, Ghanéens, Béninois, Togolais). Les femmes des pêcheurs sont impliquées dans le fumage et la livraison des produits dans l'espace Abidjan. Toutefois, les femmes autochtones des villes et villages environnants participent à ce commerce (Dabou, Jacquevilles, Grand-Lahou, Fresco, Bassam, Adiaké, Aboisso...). Cette faune aquatique, diverses et variées est composée de crevettes, d'écrevisses, de crabes et de plusieurs espèces de poissons. L'on y découvre les mêmes espèces du port, mais celle-ci sont dominées par les Ethmaloses (Ethmalosa fimbriata). les carpes (Pomadasys jubelini), les machoirons (Chrysischthys), les capitaines (Galeö̈de decadactylus), les sardinelles(Sardinella $s p$ ). Ce circuit de vente parallèle, comparativement à celui du port, a son mode de fonctionnement.

2. L'organisation de la vente

La distribution des produits de pêche est bien organisée à Abidjan. L'on retient une primauté des espaces de débarquement, de collecte et de vente des produits halieutiques (frais et fumés), les marchés de gros du poisson fumé comme le montre la figure 4 . 


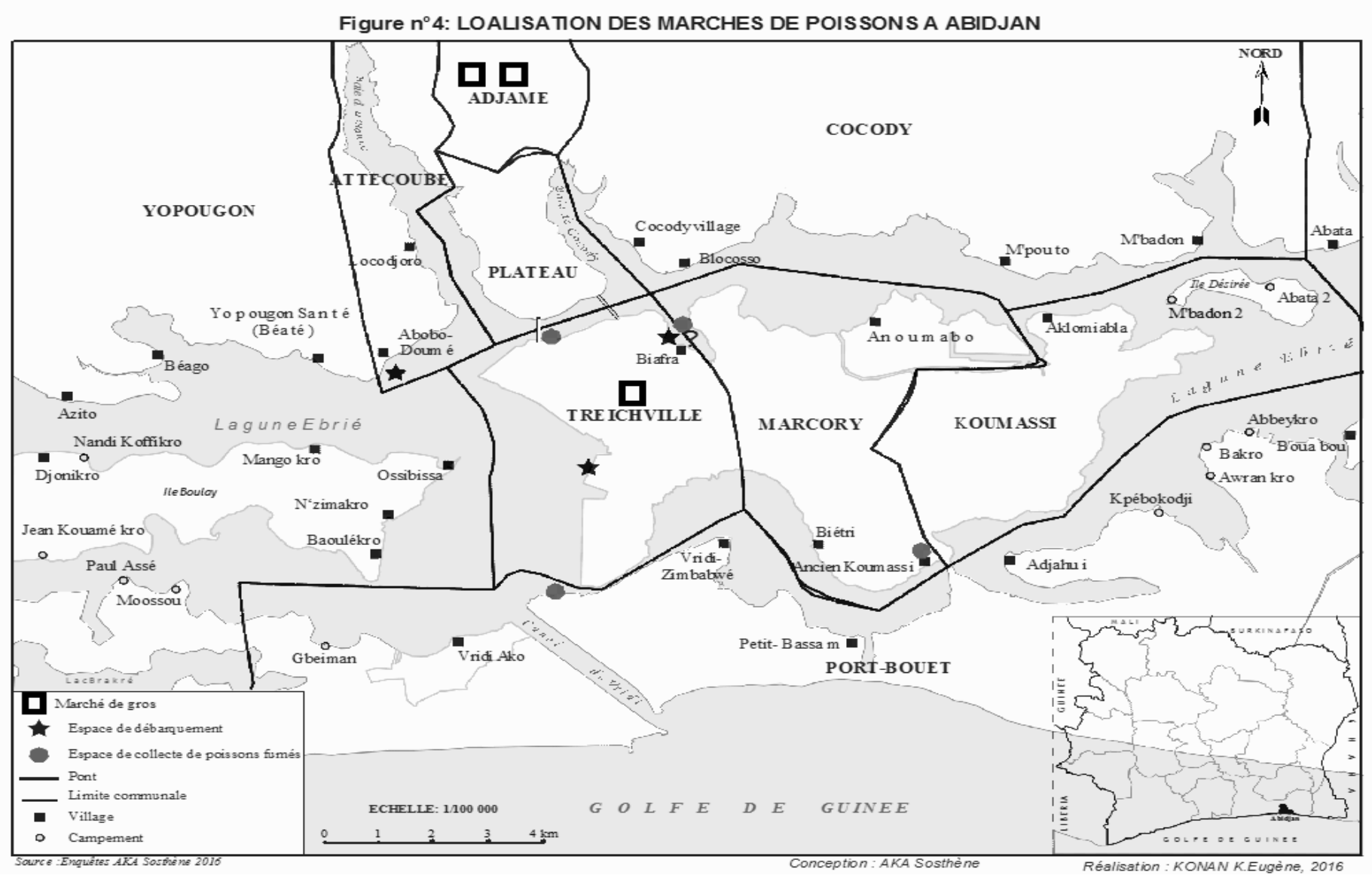


Ainsi se positionne un autre réseau de distribution, au vu de la densité hydrographique du pays. L'on peut mentionner d'autres marchés qui servent de relais comme celui de Siporex qui réceptionne le poisson frais et le poisson fumé d'Abidjan, de Jacqueville, de Grand-Lahou, de Dabou. Le grand marché de Koumassi accueille ceux de Bassam, d'Adiaké, d'Aboisso. A ces espaces de relais dans la commercialisation s'adjoignent ceux d'Abobo-doumé, du marché sicobois de Koumassi, le marché de l'académie des mers. Abobodoumé reçoit également les ressources en provenance du port. A la différence du circuit portuaire, ce sont les pinasses, les pirogues motorisées ou non qui assurent la majeure partie du transport. Il est surtout lagunaire pour desservir la ville. Les véhicules de marque Peugeot (bâchée) et les taxis communaux aident à la commercialisation. Les femmes sont très actives et sont aux différentes étapes de la vente, c'est-à-dire du grossiste au détaillant. La diffusion de ces produits permet d'atteindre tous les marchés de la ville d'Abidjan. Il existe aujourd'hui à cet effet 3 marchés de vente de gros ( grossistes) à Abidjan dont 1 à Treichville (avenue 16, rue 40) et 2 à Adjamé (le marché Gouro et celui du forum des marchés). Le marché de gros du quartier Biafra(Treichville) n 'existe plus pour des aménagements urbains. Ils sont localisés sur la figure 3. Ici, les acteurs disposent de gros paniers de 100 à $150 \mathrm{~kg}$. Le poisson, une fois acheté est regroupé dans de gros cartons. Les marchés de gros réceptionnent les mêmes marchandises et les revendent par pesées à l'aide de grandes bascules. Les commerçants disposent de grands magasins de stockage (AKA, 2006). Ils vendent certes à Abidjan, mais la majorité de la clientèle vient de l'intérieur du pays : Bondoukou, Tanda, Abengourou, Gagnoa, Daloa, Soubré, Lakota, Divo... Des producteurs aux détaillants, en passant par les grossistes, chacun semble véritablement tirer profit.

\section{Conclusion}

Le port de pêche est un maillon utile dans la chaîne des produits halieutiques du territoire ivoirien. Il marque sa particularité à Abidjan au vu de l'effort des acteurs à tous les stades de la vente quoique jalonnés de difficultés. Cette activité semble dynamique à Abidjan tenant compte de son occupation spatiale dans les différentes communes (chambres froides, marchés) pour un rapprochement des populations, dans le souci d'un ravitaillement rationnel. Dans son mode de fonctionnement, ce secteur est mieux structuré. Cependant des filières de distribution annexes dominées par les hommes existent dans ce milieu et les produits sont issus de la pêche artisanale (fleuves, lagunes, lacs, mer). Sa méthode organisationnelle vient en appoint à celle du port mêlée à une concurrence substantielle. Pour un plus grand dynamisme, le port de pêche doit renforcer sa capacité de distribution et la rendre plus accessible. 


\section{References:}

1. AKA K. S., (2006): La pêche lagunaire dans l'agglomération d'Abidjan ; mém. maîtrise, Université Cocody-Abidjan,IGT, 171p

2. CAMARA M. M. B., (2008) : Quelle gestion des pêches artisanales en Afrique de l'Ouest ; Etude de la complexité de l'espace halieutique en zone littorale sénégalaise ; Doctorat $3^{\mathrm{e}}$ Cycle, Géographie Humaine; Université CHEICK ANTA DIOP de Dakar, Sénégal ; Edition IRD, 399p.

3. CNRLTL, (2012) : Dictionnaire outils et ressources pour un traitement optimisé de la langue lexicographie, 44, avenue de la libération, bp 30687-54063 Nancy Cedex, France.

4. DAP, (2012), Annuaire des statistiques des pêches et de l'Aquaculture ; rapport d'activités Abidjan, 23p.

5. DUCRUET C., (2005): Dynamiques scalaires et temporelles des villes-ports ; typologie mondiale de 330 trajectoires urbano-portuaires, 1990-2000. Korea Research Institute for human settlements (KRIHS), Gyeonggi-do; pp 431-712, Republic of Korea.

6. I.N.S, (1975): Synthèses des résultants définitifs du Recensement Général de la Population et de 1'Habitat (RGPH), Abidjan 17p.

7. I.N.S, (2014), Synthèses des résultants définitifs du Recensement Général de la Population et de l'Habitat (RGPH), Abidjan 27p.

8. LAURIN C., (1970) : Oui, le partie Québécois vous offre solution. JL259 A56A46, Québec, Canada, 31p.

9. MIPARH, (2004) : Etude perspective pour la relance du secteur pêche et aquaculture en Côte d'Ivoire de 1990 à 2000 in rapport final volet bilan diagnostic, Coopération Belge, BENETD/Ressources animales, PP 24 -28.

10. PAA/DPP, (2002) : Projet de développement intégré au port de pêche, table ronde sur le port de pêche ; rapport final, 300p.

11. PAA, (2014) : Communauté portuaire; rapport d'activité, Edition ADA-GROUP, II plateaux vallons, Abidjan -Côte d'Ivoire, http//www.portabidjan.ci.

12. VERLAQUE, C., (1979) : Inductions portuaires : le cas Sétois ; $2^{\text {nd }}$ Colloque franco-japonais de Géographie in ville et port, développement portuaire, croissance spatiale des villes, environnement littoral; 25 septembre au 8 Octobre 1978. Paris, Editions CNRS pp $175-180$. 